\title{
Optimization of Vagal Stimulation Protocol Based on Spontaneous Breathing Rate
}

\begin{abstract}
Liliane Appratto De Souza ${ }^{1}$, Janaina Barcellos Ferreira ${ }^{2}$, Andressa Silveira de Oliveira Schein ${ }^{1}$, Daniela Ravizzoni Dartora ${ }^{1}$, Adenauer Girardi Casali ${ }^{3}$, Catharina M. Carvalho Scassola ${ }^{3}$, Eleonora Tobaldini ${ }^{4}$, Nicola Montano ${ }^{4}$, Stefano Guzzetti ${ }^{4}$, Alberto Porta ${ }^{5,6}$, Maria Claudia Irigoyen $^{1,2}$ and Karina Rabello Casali ${ }^{1,3 *}$
\end{abstract}

${ }^{1}$ Institute of Cardiology of Rio Grande do Sul, University Foundation of Cardiology, Porto Alegre, Brazil, ${ }^{2}$ Hypertension Division, Medicine School, Heart Institute, São Paulo University, São Paulo, Brazil, ${ }^{3}$ Department of Science and Technology, Institute of Science and Technology, Federal University of São Paulo, São José dos Campos, Brazil, ${ }^{4}$ Department of Clinical Science, Luigi Sacco Hospital, University of Milan, Milan, Italy, ${ }^{5}$ Department of Biomedical Sciences for Health, University of Milan, Milan, Italy, ${ }^{6}$ Department of Cardiothoracic, Vascular Anesthesia and Intensive Care, IRCCS Policlinico San Donato, San Donato Milanese, Milan, Italy

OPEN ACCESS

Edited by:

Tijana Bojić

University of Belgrade, Serbia

Reviewed by:

Eduardo Colombari,

Universidade Estadual Paulista Júlio de Mesquita Filho (UNESP), Brazil

Andreas Voss,

Institut für Innovative Gesundheitstechnologien (IGHT),

Germany

*Correspondence:

Karina Rabello Casali rabellocasali@gmail.com

Specialty section:

This article was submitted to Autonomic Neuroscience,

a section of the journal

Frontiers in Physiology

Received: 14 May 2018 Accepted: 05 September 2018 Published: 26 September 2018

Citation:

De Souza LA, Ferreira JB, Schein ASdO, Dartora DR, Casali AG,

Scassola CMC, Tobaldini E, Montano N, Guzzetti S, Porta A, Irigoyen MC and Casali KR (2018) Optimization of Vagal Stimulation

Protocol Based on Spontaneous Breathing Rate.

Front. Physiol. 9:1341. doi: 10.3389/fphys.2018.01341
Controlled breathing maneuver is being widely applied for cardiovascular autonomic control evaluation and cardiac vagal activation through reduction of breathing rate (BR). However, this maneuver presented contradictory results depending on the protocol and the chosen BR. These variations may be related to the individual intrinsic profile baseline sympathetic tonus, as described before by others. In this study, we evaluated the effect of controlled breathing maneuver on cardiovascular autonomic control in 26 healthy subjects allocated into two protocols: (1) controlled breathing in three different rates (10, 15, and 20 breaths/min) and (2) controlled breathing in rates normalized by the individual spontaneous breathing rate (SBR) at 100, 80, 70, and 50\%. Our results showed autonomic responses favorable to vagal modulation with the lower BR maneuvers. Nevertheless, while this activation was variable using the standard protocol, all participants of the normalized protocol demonstrated an increase of vagal modulation at $80 \%$ BR (HFnu $80=67.5 \%$ vs. $48.2 \%, p<0.0001$ ). These results suggest that controlled breathing protocols to induce vagal activation should consider the SBR, being limited to values moderately lower than the baseline.

Keywords: controlled breathing, autonomic nervous system, heart rate variability, spectral analysis, vagal stimulation

\section{INTRODUCTION}

Controlled breathing maneuver is a methodology widely used to evaluate cardiovascular autonomic control (Pinna et al., 2006; Adams et al., 2009; Stein et al., 2011; Porta et al., 2012; Ferreira et al., 2013; Krasnikov et al., 2013). Widespread evidence showing important effects of respiratory profile (rate, rhythm and amplitude) on beat-to-beat cardiovascular variability (Sakakibara and Hayano, 1996; Cooke et al., 1998; Bernardi et al., 2001; Pinna et al., 2006; Carnevali and Sgoifo, 2014; Laborde et al., 2017; Steffen et al., 2017) and the voluntary breathing control was proposed as an effective way to avoid confounding factors in short-time evaluations of cardiovascular variability (Radaelli et al., 1994; Pinna et al., 2006; DeBeck et al., 2010). Particularly, studies have verified that the breathing rate reduction is responsible for the increase of cardiac vagal modulation itself 
(Montano et al., 1994; Bernardi et al., 2000; Fan et al., 2011; Cabiddu et al., 2012; Tobaldini et al., 2013), which lead to the application of controlled breathing as a protocol to induce vagal activation, indicated for pathological conditions, such as hypertension (Radaelli et al., 1994; Grossman et al., 2001; Mourya et al., 2009; Gavish, 2010) and diabetes (Brown et al., 2008).

On the contrary, results associating breathing control maneuvers with lower breathing rates and the improvement of vagal modulation are still contradictory. Some studies observed an increase of cardiac sympathetic component of healthy subject in slower breathing $(0.1 \mathrm{~Hz})$ and an improvement of vagal component in faster breathing pattern $(0.2 \mathrm{~Hz})$ (Brown et al., 1993; Reimann et al., 2010). Other studies with similar protocols (controlled breathing at 0.1 and/or $0.25 \mathrm{~Hz}$ ) did not demonstrate significant changes in blood pressure variability (BPV) and heart rate variability (HRV) (Pinna et al., 2006; Tzeng et al., 2009).

A reasonable explanation for different responses to the application of the same maneuver would be the individual variability of intrinsic autonomic profiles at the baseline. A straight relation between spontaneous breathing rate (SBR) and baseline sympathetic modulation was already demonstrated (Naughton et al., 1998; Narkiewicz et al., 2006), indicating a possible association between autonomic response to the maneuver and SBR at baseline. Meanwhile, the standardization of a normalized breathing control protocol to induce vagal activity depends on a systematic investigation of the effects of controlled breathing maneuver on cardiovascular autonomic control. This standardization would be relevant not only to the evaluation of the cardiovascular autonomic control, but also to the improvement of vagal modulation as a potential therapy for many diseases (Bernardi et al., 2001; Grossman et al., 2001; Joseph et al., 2005).

In this study we applied spectral methods to evaluate the effects of controlled breathing maneuvers on cardiovascular autonomic control in healthy subjects with different breathing rates (from 6 to 22 breaths/min), fixed or normalized to spontaneous breathe. A standard protocol, based on the normalization of individual SBR, can enhance vagal activation and this standardization could improve the efficiency and clinical applicability of vagal modulation protocols.

\section{MATERIALS AND METHODS}

Twenty six healthy subjects, age between 24 and 38 years old, were enrolled into the study. Recruitment and selection were done at two clinical research centers participating in this study. The participants had not been diagnosed for hypertension, diabetes, chronic respiratory disease, autonomic dysfunctions, as well as to use tobacco, alcohol or other drugs.

All participants were requested to not ingest caffeine, alcohol or practice exhaustive physical activity $12 \mathrm{~h}$ before the protocol. The protocol was approved, according to the ethical guidelines of the 1975 Declaration of Helsinki, by the local Committee for Ethics in Research and all subjects signed an informed consent form. After fulfill inclusion criteria, the subjects were allocated in two groups: Standard Protocol (SP; $N=10$ ) or Normalized Protocol (NP; $N=16)$.

\subsection{Standard Protocol (SP)}

The subjects were evaluated in the Laboratory of Clinical Investigation, Luiggi Sacco Hospital, in a silent, controlled temperature $\left( \pm 23^{\circ} \mathrm{C}\right)$ and illumination ambient. The electrocardiogram (ECG) was recorded at $300 \mathrm{~Hz}$ and the pulse pressure signal was continuously and noninvasively acquired at 1,000 Hz, in the supine position (Finapres 2300, Ohmeda, Englewood, CO). The participants of the SP were asked to breath at specific breathing rates usually found in the literature. First, the subjects should stay at rest, breathing spontaneously for $10 \mathrm{~min}$ while ECG would be registered. Afterwards, they should breathe following the sound generated by a digital metronome, to control breathing at different rates: $0.17 \mathrm{~Hz}$ (10 breathe/min, R10), $0.25 \mathrm{~Hz}$ (15 breathe/min, R15) e $0.33 \mathrm{~Hz}$ (20 breathe/min, R20). The blocks R10, R15 and R20 were set randomly. A 10 -min register was performed for each protocol, with a 5 min interval between each other.

\subsection{Normalized Protocol (NP)}

The subjects were evaluated in the Laboratory of Clinical Investigation, Cardiology Institute, in a silent, controlled temperature $\left( \pm 23^{\circ} \mathrm{C}\right)$ and illumination ambient. The pulse pressure signal was continuously and noninvasively acquired at $1,000 \mathrm{~Hz}$, in the supine position (Finapres 2300, Ohmeda, Englewood, CO, United States).

In the Normalized Protocol, controlled breathing maneuvers were determined by normalization of individual SBR. First, the subjects were asked to be at rest for $10 \mathrm{~min}$, while BP signals were acquired, and the SBR was observed. Afterwards, they should follow the sound generated by a digital metronome, based on individual observation, set randomly: $100,80,70,60$, and $50 \%$ of SBR. A 10-min register was acquired for each protocol, with a 5 min interval between each other.

\subsection{Autonomic Control Assessment and Evaluation}

In the SP, the time series of heart rate (tachogram) were obtained from the interval between two consecutive peaks of RR interval (ECG records). In the NP, the time series of heart rate were generated by detection of the systolic peaks records of blood pressure. Stationary sequences of 200-300 beats, and coincident in both protocols, were selected (Porta et al., 2004). Frequency domain analysis of HRV and BPV was performed with an autoregressive algorithms, with emphasis on very low frequency (VLF: 0.00-0.04 Hz), low frequency (LF: 0.04-0.15 Hz) and high frequency (HF: 0.15-0.40 Hz) bands.

The spectral components were expressed in absolute (abs) and normalized units (nu). In the HRV spectrum LF and HF bands represent sympathetic and parasympathetic modulation, respectively. The ratio between $\mathrm{LF} / \mathrm{HF}$ components is related to the cardiac sympathovagal balance (Malliani et al., 1991; Montano et al., 2009). Regarding the systolic blood pressure variability, the absolute LF component value represents the sympathetic vascular modulation (Stauss, 2007). The relation 
between the LF component of the heart rate and systolic blood pressure variability corresponds to the spontaneous baroreflex sensitivity ( $\alpha_{\text {LF }}$ index) (Fazan et al., 2005).

Time domain indices of HRV quantify the variability between successive beats. SDNN index (ms) represents the standard deviation of normal-to-normal (NN) heart beats. The pNN50 index corresponds to percentage of adjacent NN intervals that differ from each other by more than $50 \mathrm{~ms}$. The RMSSD index is calculated by the root mean square of successive differences between normal heart beats (RMSSD).

\subsection{Statistical Analysis}

Data are shown as mean \pm standard deviation. Statistical analyses were performed using one-way ANOVA followed by Tukey post-hoc test. For nonparametric data Friedman ANOVA test with repeated measures was applied. Pearson correlation was used to assess the association among variables. $P<0.05$ was considered significant. Significant effects of the controlled breathing protocol on HRV parameters were accessed by a Linear Mixed Model (LMM). Estimation of fixed effects and covariance parameters was performed using the Restricted Maximum Likelihood (ReML) method. Null hypotheses were tested using Type III F-statistics and rejected if $p<0.05$. Main effects of multiple-levels factors were compared using Bonferroni's adjustment of confidence intervals. Dependent variables were modeled including a fixed factor associated to the group (basal, $100,80,70,60$, and 50\%), a random factor associated with the intercept for each subject, in order to handle the unbalanced repeated measures, and a random subject-specific effect of the respiratory maneuver. This additional random factor allows the variance of dependent variables to differ across groups. Finally, residual covariances were assumed diagonal and homogeneous.

\section{RESULTS}

\subsection{Standard Protocol (SP)}

Ten healthy volunteers, age between 24 and 32 years old, were recruited and allocated into the SP. The subjects had a mean $\mathrm{SBR}=14.5 \pm 1.7$ breathes $/ \mathrm{min}$. Statistical analysis between the groups of men (four) and women (six) showed no significant differences in autonomic parameters. The maneuvers did not induce changes in the values of HR and SBP (Table 1). In time domain analysis of HRV, SDNN index was reduced in R20 block. However, the assessment of autonomic control through spectral analysis showed significant changes in HRV, especially in the R10 ventilation block. Although the total spectral power value, which reports the value of HRV, was not changed, the maneuver in 10 breathe/min evoked changes related to the spectral components with increased HF band, compared to Basal, R15 and R20 blocks, both in absolute ( $p=0.022, p=0.015$, and $p=0.011$, respectively), and in normalized ( $p=0.041$ ) values (Figure 1). In addition, the LF/HF ratio, which is related to sympathetic-vagal balance, was lower in the R10 block in comparison to R15 and R20 ( $p$ $=0.028$ ) blocks. There was no change in the parameters of VPA and its spectral components, as well as in the $\alpha$ index, related to spontaneous baroreflex sensitivity.

The results indicated in Figure 2 shows the effect of controlled ventilation on the HFnu component for each subject in all different blocks of the SP. The protocol induced an increase of vagal activation in $80 \%$ of individuals R10 block (Figure 2A) in 50\% R15 block (Figure 2B) and $40 \%$ of those of R20 block (Figure 2C).

\subsection{Normalized Protocol (NP)}

Sixteen individuals, age between 24 and 38 years old, were recruited and allocated into the NP. The subjects studied in NP protocol presents an average $\mathrm{SBR}=16.3 \pm 2.5$ breaths $/ \mathrm{min}$. Statistical analysis between the groups of men (six) and women (10) showed no significant differences in autonomic parameters. Blocks with imposing percentage of respiratory frequencies lower than the SBR generated displacement of HF spectral component toward the LF range. Statistical analysis between SP and NP groups at rest, showed no significant differences in autonomic parameters. Figure 3 shows the spectra of HRV and respiratory signal from one individual under situations of controlled ventilation to $100 \%$ (Figure 3A), 70\% (Figure 3B), and $50 \%$ (Figure 3C) of the SBR, showing the displacement of greater coherence between the spectral band components, according to the imposed FR. Only for 100 and $80 \%$ ranges, the FR remained within the HF band in all subjects. When there is such an overlap of sympathetic and vagal frequency bands, the spectral analysis is not able to quantify both modulations apart (Malliani et al., 1991; Radaelli et al., 1994; Bernardi et al., 2000). Therefore, the results of autonomic control assessment obtained by spectral analysis are shown only for those individuals in which there was no overlap of the spectral bands while controlled ventilation maneuver was applied (Figures 4, 5 and Table 2).

There were no changes in HR and SBP, as well as in the values of HRV and BPV components. The assessment of autonomic control demonstrated changes in the LF and HF components, related to sympathetic and cardiac vagal modulation, respectively (Table 2, Figures 4A,B, 5). Furthermore, the ventilation control lead to increase of HFnu component $(p<0.0001)$, reduction of LFnu component $(p<0.0001)$ and of LF/HF index $(p=$ 0.01 ) of all VC sessions in relation to baseline. There were no changes in the spectral BPV components for any sessions to which subjects underwent. However, when evaluated individually it was observed that the responses did not occur homogeneously in every situation.

The effect of the NP on the HFnu component of all ventilation blocks for each subject individually is observed in Figure 5. The protocol induced an increase of vagal activation or no changes of HFnu component in $81 \%$ of subjects underwent to controlled ventilation in SBR (Figure 5A). On the $80 \%$ block ventilation of SBR, all subjects showed such results (Figure 5B). In addition, a total of $81 \%$ of subjects responded with activation of vagal modulation or no change in the component HFnu block ventilation to $70 \%$ of RES (Figure 5C) as well as half of the subjects of the ventilating block $60 \%$ of the SBR (Figure 5D) responded similarly only $37 \%$ of subjects subjected to block ventilation of $50 \%$.

\section{DISCUSSION}

The controlled ventilation maneuver lead to vagal activation and it is indicated in the treatment of hypertension, in 
TABLE 1 | Autonomic cardiovascular control of standard protocol group.

\begin{tabular}{|c|c|c|c|c|c|}
\hline & Basal & R10 & R15 & R20 & $p$ \\
\hline HR (bpm) & $60.69 \pm 10.92$ & $60.03 \pm 11.95$ & $59.79 \pm 11.17$ & $59.43 \pm 11.04$ & 0.83 \\
\hline SAP (mmHg) & $104.86 \pm 16.04$ & $102.57 \pm 8.82$ & $101.22 \pm 13.4$ & $104.19 \pm 9.48$ & 0.78 \\
\hline SDNN (mss) & $57.46 \pm 27.06$ & $57.46 \pm 27.03$ & $52.09 \pm 18.27$ & $45.62 \pm 15.65^{\star} \#$ & 0.01 \\
\hline RMSSD (ms) & $54.82 \pm 30.60$ & $54.82 \pm 30.60$ & $52.08 \pm 24.93$ & $46.69 \pm 24.30$ & 0.31 \\
\hline pNN50(ms) & $32.35 \pm 24.64$ & $32.35 \pm 2.64$ & $32.18 \pm 24.36$ & $24.77 \pm 21.83$ & 0.24 \\
\hline \multicolumn{6}{|l|}{ Spectral analysis } \\
\hline $\mathrm{HRV}\left(\mathrm{ms}^{2}\right)$ & 1786.18 (1057.9-4281.95) & 2959.62 (1619.57-4794.65) & $2797.81(1364.5-5163.25)$ & 1957.86 (880.13-3382.84) & 0.45 \\
\hline $\mathrm{LFa}\left(\mathrm{ms}^{2}\right)$ & $493.16(246.3-1473.93)$ & 434.51 (161.06-1004.96) & 452.55 (192.82-2266.79) & 518.67 (264.83-646.21) & 0.23 \\
\hline LF nu & 43.44 (33.73-51.33) & $31.80(9.87-4.21)^{\star}$ & $46.42(21.98-84.65)^{\#}$ & $54.62(34.18-63.49)^{\#}$ & 0.03 \\
\hline $\mathrm{HFa}\left(\mathrm{ms}^{2}\right)$ & $1093.92 \pm 1054.26$ & $1832.73 \pm 1610.46^{\star}$ & $920.37 \pm 1098.35^{\#}$ & $804.46 \pm 773.96^{\#}$ & 0.01 \\
\hline HF nu & $55.71(38.30-59.01)$ & $66.87(46.10-88.53)^{*}$ & $45.98(13.62-76.12)^{\#}$ & $43.81(33.81-61.05)^{\#}$ & 0.04 \\
\hline SAPV $\left(\mathrm{mmHg}^{2}\right)$ & 13.04 (5.46-21.23) & $12.53(6.1-46.67)$ & 10.25 (4.37-24.95) & $9.06(2.45-17.31)$ & 0.49 \\
\hline LFabs $\left(m m g^{2}\right)$ & $1.63 \pm 1.10$ & $5.57 \pm 6.48$ & $5.52 \pm 5.69$ & $2.32 \pm 2.72$ & 0.09 \\
\hline HFabs $\left(m m H^{2}\right)$ & $0.98(0.5-1.37)$ & $1.98(1.4-3.81)$ & $0.63(0.32-0.93)$ & $0.61(0.46-1.05)$ & 0.01 \\
\hline$\alpha$-index & $26.63 \pm 20.99$ & $14.65 \pm 11.37$ & $27.98 \pm 25.36$ & $31.20 \pm 24.63$ & 0.14 \\
\hline
\end{tabular}

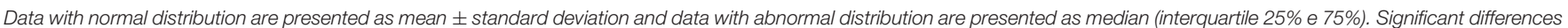

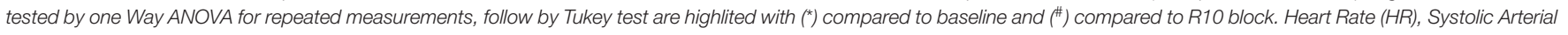

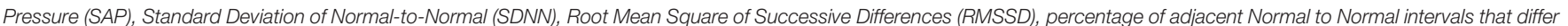

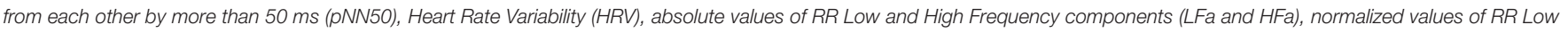

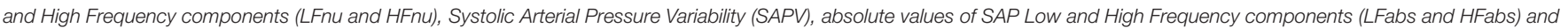
barorreflex index $(\alpha$-index). Signficant $(p<0.05) p$-values are displayed in bold face.

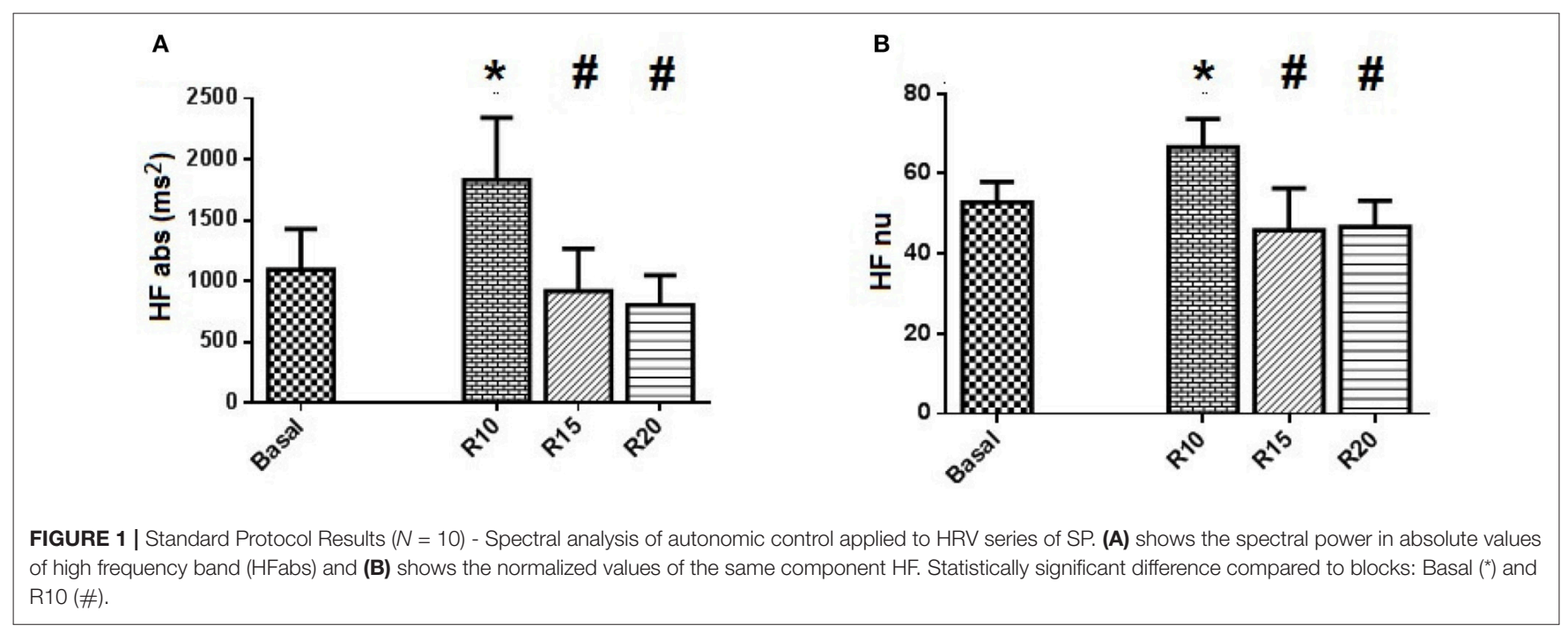

order to reduce blood pressure and improve the clinical profile (Radaelli et al., 1994; Sakakibara and Hayano, 1996; Cooke et al., 1998; Grossman et al., 2001; Pinna et al., 2006; Reimann et al., 2010). Several studies, however, consider absolute respiratory frequencies of 10 or 15 breaths/min to be applied indiscriminately in many situations. The respiratory rate indicated by the Brazilian Guidelines of Hypertension, for example, is 10 breaths/min (SocBrasCardio, 2010).

Our results highlight for the very first time the importance of considering individual spontaneous respiratory rate and its effect on basal sympathetic tone and vagal activation. Although we observed a significant increase in vagal activation at 10 breaths/min (Figure 1), the individual analysis of subjects treated with the SP (Figure 2) shows the heterogeneity of the responses within this group, with increased vagal activation in $80 \%$ of subjects in R10, $50 \%$ of R15 individuals and $40 \%$ of those of R20. When we take into account the individual SBR and apply the normalized protocol, vagal modulation was already increased in $81 \%$ of subjects just by controlling the ventilation at individual SBRs (Figure 5A). Moreover, vagal activation was achieved with homogeneity in all individuals when breathing at a rate of $80 \%$ SBR (Figure 5B). 


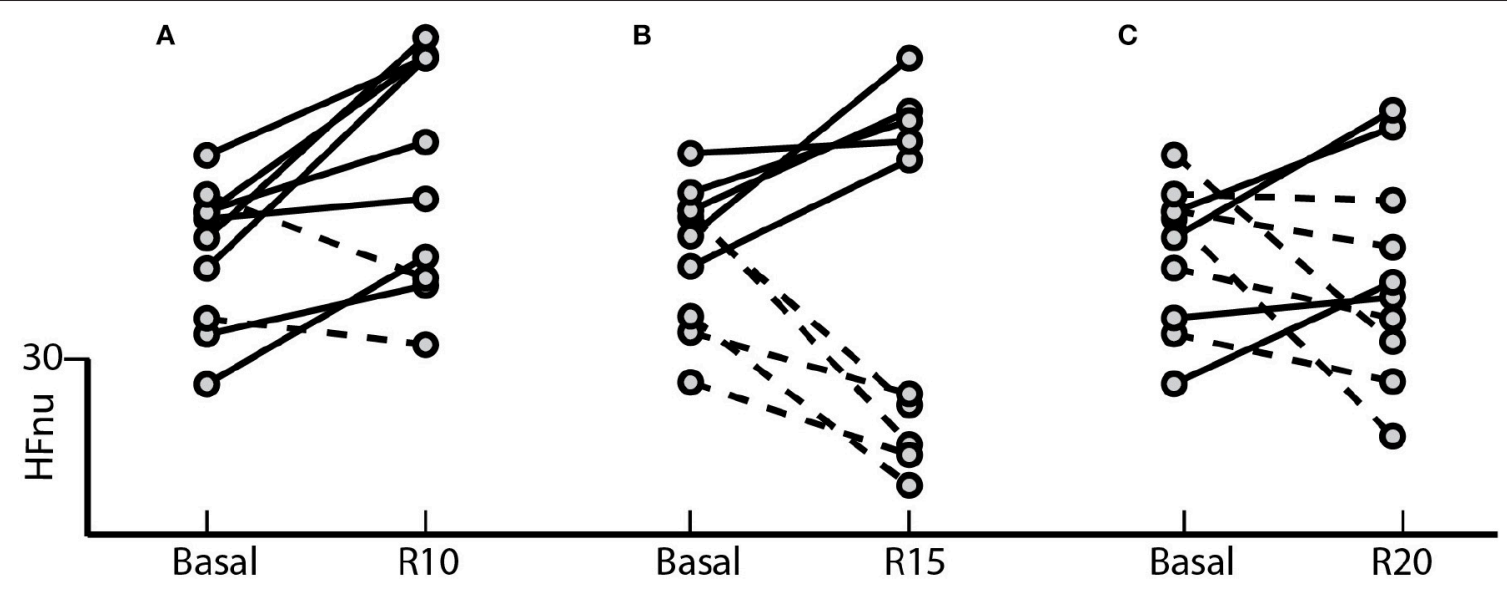

FIGURE 2 | Standard Protocol Results ( $N=10$ ) - Effect of controlled ventilation maneuvers on the normalized HF component (HFnu) in subjects treated with SP. Solid lines denote transitions with increased HF component with respect to the baseline during the maneuver controlled ventilation. The dotted lines marked decrease in this component during the maneuver. The protocol induced an increase in HFnu on (A) 8 of 10 subjects in R10, (B) 5 of 10 in R15 and (C) 4 of 10 in R20.
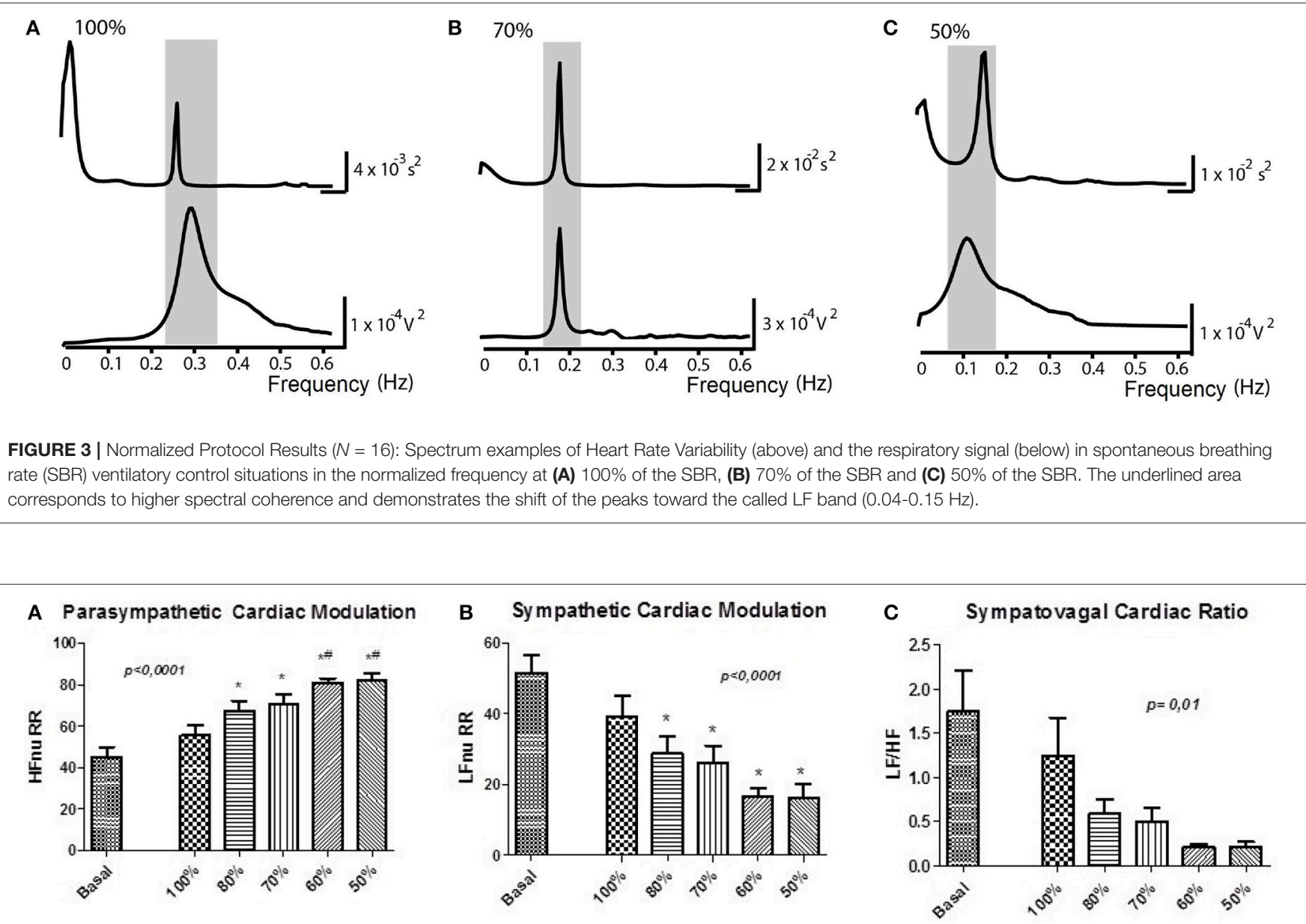

FIGURE 4 | Normalized Protocol Results ( $N=16)$ : Parameters for the assessment of autonomic control through spectral analysis applied to the pulse interval series of NP. (A) shows the normalized spectral power of the high frequency HF (HFnu) band, (B) shows the normalized spectral power of the low frequency band LF ( $\mathrm{LFnu}$ ) and (C) shows the relationship between the spectral bands LF/HF. Statistically significant difference compared to blocks: Basal $\left(^{\star}\right)$ and $100 \%$ (\#). 

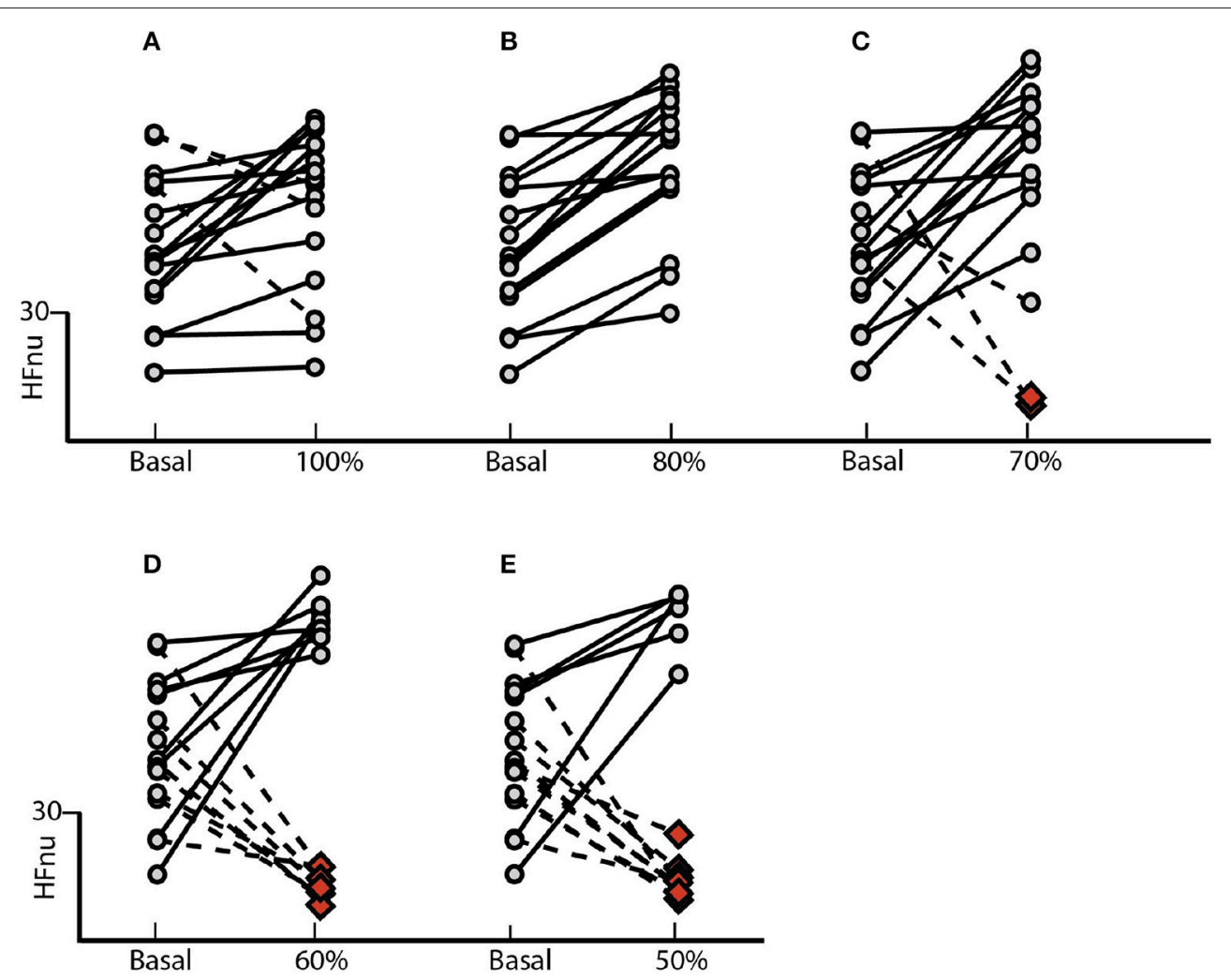

FIGURE 5 | Normalized Protocol Results $(N=16)$ : Effect of controlled ventilation maneuver on the normalized HF component (HFnu) in subjects treated with NP. Solid lines denote transitions with increased HF component when compared with baseline. The dotted lines marked decrease in this component. The protocol induced an increase or no change in the HF component (A) on 13 of 16 individuals into the group 100\%, (B) in all 16 subjects into the group $80 \%$, (C) in 13 of 16 individuals into the group $70 \%$, (D) in 8 of 16 individuals into the group $60 \%$ and (E) in 6 of 16 individuals into the group 50\%. The red dots indicate the spectral overlap of the HF band on the LF, blocking the application of such methodology.

It is known that slow breathing induces a generalized decrease of excitatory pathways in the regulation of cardiovascular and respiratory systems (Joseph et al., 2005; Laborde et al., 2017; Steffen et al., 2017). Some authors have shown that controlled ventilation, particularly slow breathing 3-6 breaths/min, reduces $\mathrm{HR}$ and BP both in healthy individuals (Sakakibara and Hayano, 1996; Krasnikov et al., 2013) and hypertensive patients (Joseph et al., 2005). The mechanisms involved in the changes imposed by slow ventilation, however, are not yet fully known. Some authors attribute these effects to reflex changes, such as increased baroreflex sensitivity (Bernardi et al., 2001; Joseph et al., 2005; Reimann et al., 2010) and reduction in chemoreflex. Anatomical changes of pulmonary distress may also evoke autonomic responses (Bernardi et al., 2001; Cabiddu et al., 2012) and the Hering-Breuer reflex, which induces inhibition of inspiration after inflation of the lung, is also mediated by vagal innervation and appears to have an important role in the regulation of respiration and respiratory depth (Clark and von Euler, 1972). Our results showed no changes in spontaneous baroreflex sensitivity, which can be attributed to an indirect evaluation method (Fazan et al., 2005) or to methodological variations (Tzeng et al., 2009). In any event, our results suggest that the order and magnitude of these possible mechanisms of action during the operation of controlled ventilation depend on individual characteristics such as spontaneous breathing frequency. In the group to which it was imposed a fixed respiratory rate of $10 \mathrm{breaths} / \mathrm{min}$, we could find individuals breathing at their own SBR and others breathing at a rate near $50 \%$ of their SBR. Our study supports the hypothesis that this maneuver have different effects in the two cases: in these latter individuals such a maneuver would not only be neffective in reducing $\mathrm{BP}$, but could also evoke sympathetic activation and reduce vagal modulation.

Recent study evaluated the effects of gradual increase and decrease of breathing rate on healthy subjects and appointed that, in the few subjects who had significant trends, sympathetic burst areas could change directly or inversely with breathing frequency (Stankovski et al., 2013).

Protocols that impose very slow breathing rates can induce physiological changes in lung function in search of adaptation to prolonged ventilation. Such changes may occur acutely and are deleted when the protocol involves training with evaluation of chronic effects. Both hypoxia and hypercapnia increase ventilation and can increase sympathetic tone, HR and SBP (Somers et al., 1989a,b; Van de Borne et al., 2000). Our study did not evaluate the parameters of respiratory function due to the fact that the methodology be able to insert autonomic changes confounding the analysis (Bernardi et al., 2000). 
TABLE 2 | Autonomic Cardiovascular Control of Normalized Protocol Group.

\begin{tabular}{|c|c|c|c|c|c|c|c|}
\hline & Basal & $100 \%$ & $80 \%$ & $70 \%$ & $60 \%$ & $50 \%$ & $\mathbf{p}$ \\
\hline HR (bpm) & $70 \pm 10$ & $70 \pm 11$ & $69 \pm 10$ & $70 \pm 10$ & $70 \pm 10$ & $70 \pm 9$ & 0.95 \\
\hline SAP (mmHg) & $116 \pm 14$ & $114 \pm 14$ & $118 \pm 15$ & $119 \pm 14$ & $117 \pm 15$ & $115 \pm 16$ & 0.28 \\
\hline SDNN (mss) & $50.96 \pm 21.98$ & $53.89 \pm 37.97$ & $57.01 \pm 30.91$ & $58.63 \pm 27.44$ & $62.25 \pm 31.42$ & $60.46 \pm 16.57$ & 0.07 \\
\hline RMSSD (ms) & $53.87 \pm 39.21$ & $50.98 \pm 39.79$ & $53.57 \pm 39.18$ & $51.21 \pm 32.2$ & $53.58 \pm 35.84$ & $55.58 \pm 39.72$ & 0.27 \\
\hline pNN50(ms) & $23.51 \pm 22.47$ & $24.13 \pm 25.05$ & $28.45 \pm 24.09$ & $26.43 \pm 22.1$ & $26.99 \pm 23.35$ & $27.18 \pm 20.32$ & 0.34 \\
\hline \multicolumn{8}{|l|}{ Spectral analysis } \\
\hline$N$ & 16 & 16 & 16 & 14 & 8 & 6 & \\
\hline $\mathrm{HRV}\left(\mathrm{ms}^{2}\right)$ & $2301 \pm 1931$ & $2603 \pm 2157$ & $2877 \pm 2008$ & $3108 \pm 2236$ & $4076 \pm 2914$ & $3461 \pm 1105$ & 0.61 \\
\hline VLFa $\left(\mathrm{ms}^{2}\right)$ & $387.07 \pm 447.81$ & $311.87 \pm 366.88$ & $421.33 \pm 471.73$ & $465.91 \pm 469.59$ & $510.15 \pm 706.83$ & $547.1 \pm 351.51$ & 0.29 \\
\hline $\mathrm{LFa}\left(\mathrm{ms}^{2}\right)$ & $875 \pm 867$ & $953 \pm 1309$ & $605 \pm 483$ & $553 \pm 527$ & $489 \pm 285$ & $427 \pm 231$ & 0.34 \\
\hline LF nu & $48.5 \pm 19.4$ & $39.2 \pm 23.3$ & $28.6 \pm 19.4^{\star}$ & $26.2 \pm 17.6^{\star}$ & $16.8 \pm 6.4^{\star \#}$ & $16.4 \pm 9.2^{\star \#}$ & $<0.0001$ \\
\hline $\mathrm{HFa}\left(\mathrm{ms}^{2}\right)$ & $976 \pm 998$ & $1253 \pm 1203$ & $1759 \pm 1479$ & $1992 \pm 1768$ & $2817 \pm 2109$ & $2292 \pm 1022$ & 0.05 \\
\hline HF nu & $48.2 \pm 18.7$ & $55.4 \pm 20.8$ & $67.5 \pm 18.8^{\star}$ & $70.4 \pm 18.1^{\star \#}$ & $81 \pm 6.4^{\star \#}$ & $82.3 \pm 8.7^{\star \#}$ & $<0.0001$ \\
\hline LF/HF & $1.62 \pm 1.85$ & $1.25 \pm 1.72$ & $0.59 \pm 0.67^{\star}$ & $0.50 \pm 0.58^{*}$ & $0.21 \pm 0.09^{\star}$ & $0.21 \pm 0.15$ & 0.01 \\
\hline SAPV $\left(m m H g^{2}\right)$ & $23.2 \pm 24.0$ & $22.1 \pm 17.3$ & $26.9 \pm 24.9$ & $30.8 \pm 30.5$ & $28.3 \pm 17.8$ & $42.1 \pm 37.0$ & 0.47 \\
\hline LFabs $\left(m m H^{2}\right)$ & $5.6 \pm 12.5$ & $6.9 \pm 9.6$ & $15.4 \pm 25.6$ & $9.6 \pm 14.9$ & $12.9 \pm 17.4$ & $18.0 \pm 21.0$ & 0.32 \\
\hline$\alpha$-index & $12.6 \pm 5.5$ & $15.2 \pm 12.0$ & $14.5 \pm 11.1$ & $13.4 \pm 11.7$ & $14.6 \pm 17.8$ & $13.2 \pm 16.7$ & 0.69 \\
\hline
\end{tabular}

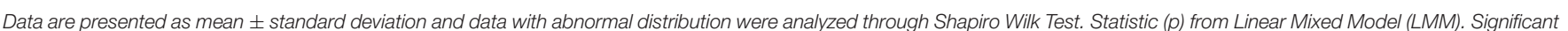

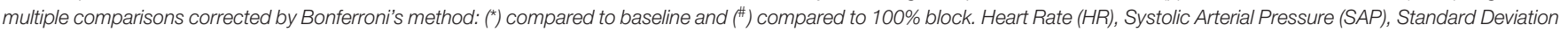

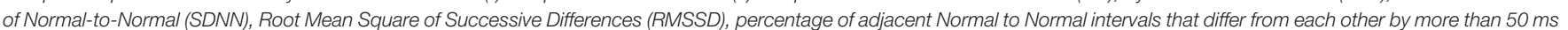

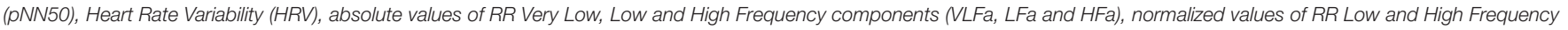

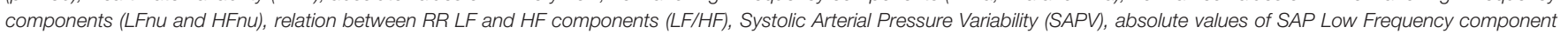
(LFabs) and barorreflex index ( $\alpha$-index). Signficant $(p<0.05) p$-values are displayed in bold face.

A possible increase in cardiac sympathetic component has importance both as investigative clinical character once controlled ventilation has been applied as drug intervention in several diseases such as in hypertension (Radaelli et al., 1994; Grossman et al., 2001; Mourya et al., 2009; Gavish, 2010), diabetes (Brown et al., 2008) and acute myocardial infarction (Adams et al., 2009). Abnormal respiratory modulation is often related to autonomic dysfunction and manipulation of the breathing pattern can promote beneficial effects for the cardiovascular and respiratory control in both physiological and pathological conditions effects. Thus, it opens a new field for future research aiming at improving the management of patients with cardiovascular autonomic dysfunction (Bernardi et al., 2001). However, the success of the method depends largely on the interaction of the patient and the pathological conditions (Gavish, 2010).

\section{CONCLUSION}

In conclusion, we have shown the importance of SBR in the evaluation for the application of maneuvers controlled ventilation. We demonstrate the behavior of the autonomic control with the variation of RF, by proposing a standard baseline for each subject protocol. The use of the maneuver of controlled ventilation at $80 \%$ of SBR evoked increase in cardiac vagal component in all subjects assessed, enhancing its application in protocols designed to improve vagal activation in healthy individuals, in view of the importance of applying a method with known effect and the most reliable possible, and motivating the study of such a protocol in pathological situations.

\section{OUTLOOK}

As perspectives, the standard protocol could be applied to populations of different pathologies for both assessments for chronic and acute effect study by adaptively training, seeking the construction of individual models for more efficient responses. Furthermore, the study of the acute effect of slow BR with methodological rigor, evaluating directly the sympathetic tone and respiratory function, could provide more precise information about the effect of FR at $50 \%$ of SBR on cardiovascular autonomic control.

\section{LIMITATIONS}

We admit as an important limitation of this study, the lack of respiratory function evaluation, such as spirometry. We understand that this evaluation could lead to more detailed analysis on the possible physiological adaptations during maneuvers. In addition, we could be more accurate in the HRV analysis if we also had ECG records.

\section{AUTHOR CONTRIBUTIONS}

KC, MI, and NM conceived and designed this study. LD, JF, AS, DD, ET, SG, and AP collected the data and LD, and AC analyzed 
it. LS, AC, CS, and KC drafted the article. DD, AC, CS, NM, $\mathrm{MI}$, and $\mathrm{KC}$ made the critical revision of the article. All authors approved the final version.

\section{FUNDING}

This work was supported by the São Paulo Research Foundation (FAPESP, Brazil), National Council for Scientific and Technological Development (CNPq), Coordination

\section{REFERENCES}

Adams, J., Julian, P., Hubbard, M., Hartman, J., Baugh, S., Segrest, W., et al. (2009). A randomized controlled trial of a controlled breathing protocol on heart rate variability following myocardial infarction or coronary artery bypass graft surgery. Clin. Rehabil. 23, 782-789. doi: 10.1177/0269215509334834

Bernardi, L., Porta, C., Gabutti, A., Spicuzza, L., and Sleight, P. (2001). Modulatory effects of respiration. Auton. Neurosci. 90, 47-56. doi: 10.1016/S1566-0702(01)00267-3

Bernardi, L., Wdowczyk-Szulc, J., Valenti, C., Castoldi, S., Passino, C., Spadacini, G., et al. (2000). Effects of controlled breathing, mental activity and mental stress with or without verbalization on heart rate variability. J. Am. Coll. Cardiol. 35, 1462-1469. doi: 10.1016/S0735-1097(00)00595-7

Brown, C. M., Marthol, H., Zikeli, U., Ziegler, D., and Hilz, M. (2008). A simple deep breathing test reveals altered cerebral autoregulation in type 2 diabetic patients. Diabetologia 51, 756-761. doi: 10.1007/s00125-008-0958-3

Brown, T. E., Beightol, L. A., Koh, J., and Eckberg, D. L. (1993). Important influence of respiration on human $\mathrm{rr}$ interval power spectra is largely ignored. J. Appl. Physiol. 75, 2310-2317. doi: 10.1152/jappl.1993.75.5.2310

Cabiddu, R., Cerutti, S., Werner, S., Viardot, G., and Bianchi, A. M. (2012). Modulation of the sympatho-vagal balance during sleep: frequency domain study of heart rate variability and respiration. Front. Physiol. 3:45. doi: 10.3389/fphys.2012.00045

Carnevali, L., and Sgoifo, A. (2014). Vagal modulation of resting heart rate in rats: the role of stress, psychosocial factors, and physical exercise. Front. Physiol. 5:118. doi: 10.3389/fphys.2014.00118

Clark, F., and von Euler, C. v. (1972). On the regulation of depth and rate of breathing. J. Physiol. 222, 267-295. doi: 10.1113/jphysiol.1972.sp009797

Cooke, W. H., Cox, J. F., Diedrich, A. M., Taylor, J. A., Beightol, L. A., Ames, J. E., et al. (1998). Controlled breathing protocols probe human autonomic cardiovascular rhythms. Am. J. Physiol. Heart Circ. Physiol. 274, H709-H718. doi: 10.1152/ajpheart.1998.274.2.H709

DeBeck, L. D., Petersen, S. R., Jones, K. E., and Stickland, M. K. (2010). Heart rate variability and muscle sympathetic nerve activity response to acute stress: the effect of breathing. Am. J. Physiol. Regul. Integr. Comp. Physiol. 299, R80-R91. doi: 10.1152/ajpregu.00246.2009

Fan, W.-H., Ko, J.-H., Lee, M.-J., Xu, G., and Lee, G.-S. (2011). Response of nasal airway and heart rate variability to controlled nasal breathing. Eur. Arch. Otorhinolaryngol. 268, 547-553. doi: 10.1007/s00405-010-1402-0

Fazan, R.Jr., de Oliveira, M., Dias da Silva, V. J., Joaquim, L. F., Montano, N., Porta, A., et al. (2005). Frequency-dependent baroreflex modulation of blood pressure and heart rate variability in conscious mice. Am. J. Physiol. Heart Circ. Physiol. 289, H1968-H1975. doi: 10.1152/ajpheart.01224.2004

Ferreira, J. B., Plentz, R. D. M., Stein, C., Casali, K. R., Arena, R., and Dal Lago, P. (2013). Inspiratory muscle training reduces blood pressure and sympathetic activity in hypertensive patients: a randomized controlled trial. Int. J. Cardiol. 166, 61-67. doi: 10.1016/j.ijcard.2011.09.069

Gavish, B. (2010). Device-guided breathing in the home setting: technology, performance and clinical outcomes. Biol. Psychol. 84, 150-156. doi: 10.1016/j.biopsycho.2010.02.013

Grossman, E., Grossman, A., Schein, M., Zimlichman, R., and Gavish, B. (2001). Breathing-control lowers blood pressure. J. Hum. Hypertens. 15, 263. doi: $10.1038 /$ sj.jhh. 1001147 for the Improvement of Higher Education Personnel (CAPES) and Rio Grande do Sul Research Foundation (FAPERGS).

\section{ACKNOWLEDGMENTS}

To the research group in physiology from UFCSPA, especially the Prof. Dra. Katya Vianna Rigatto, Prof. Dr. Rodrigo Della Méa Plentz and Ph.D. student Cinara Stein.
Joseph, C. N., Porta, C., Casucci, G., Casiraghi, N., Maffeis, M., Rossi, M. et al. (2005). Slow breathing improves arterial baroreflex sensitivity and decreases blood pressure in essential hypertension. Hypertension 46, 714-718. doi: 10.1161/01.HYP.0000179581.68566.7d

Krasnikov, G. V., Tyurina, M. Y., Tankanag, A. V., Piskunova, G. M., and Chemeris, N. K. (2013). Analysis of heart rate variability and skin blood flow oscillations under deep controlled breathing. Respir. Physiol. Neurobiol. 185, 562-570. doi: 10.1016/j.resp.2012.11.007

Laborde, S., Mosley, E., and Thayer, J. F. (2017). Heart rate variability and cardiac vagal tone in psychophysiological research-recommendations for experiment planning, data analysis, and data reporting. Front. Psychol. 8:213. doi: 10.3389/fpsyg.2017.00213

Malliani, A., Pagani, M., Lombardi, F., and Cerutti, S. (1991). Cardiovascular neural regulation explored in the frequency domain. Circulation 84, 482-492. doi: 10.1161/01.CIR.84.2.482

Montano, N., Porta, A., Cogliati, C., Costantino, G., Tobaldini, E., Casali, K. R., et al. (2009). Heart rate variability explored in the frequency domain: a tool to investigate the link between heart and behavior. Neurosci. Biobehav. Rev. 33, 71-80. doi: 10.1016/j.neubiorev.2008.07.006

Montano, N., Ruscone, T. G., Porta, A., Lombardi, F., Pagani, M., and Malliani, A. (1994). Power spectrum analysis of heart rate variability to assess the changes in sympathovagal balance during graded orthostatic tilt. Circulation 90, 1826-1831. doi: 10.1161/01.CIR.90.4.1826

Mourya, M., Mahajan, A. S., Singh, N. P., and Jain, A. K. (2009). Effect of slow-and fast-breathing exercises on autonomic functions in patients with essential hypertension. J. Altern. Complementary Med. 15, 711-717. doi: 10.1089/acm.2008.0609

Narkiewicz, K., Van De Borne, P., Montano, N., Hering, D., Kara, T., and Somers, V. K. (2006). Sympathetic neural outflow and chemoreflex sensitivity are related to spontaneous breathing rate in normal men. Hypertension 47, 51-55. doi: 10.1161/01.HYP.0000197613.47649.02

Naughton, M. T., John, S., Rahman, M. A., Jamal, M., and Bradley, T. D. (1998). Respiratory correlates of muscle sympathetic nerve activity in heart failure. Clin. Sci. 95, 277-285. doi: 10.1042/cs0950277

Pinna, G. D., Maestri, R., La Rovere, M. T., Gobbi, E., and Fanfulla, F. (2006). Effect of paced breathing on ventilatory and cardiovascular variability parameters during short-term investigations of autonomic function. Am. J. Physiol. Heart Circ. Physiol. 290, H424-H433. doi: 10.1152/ajpheart.00438.2005

Porta, A., Bassani, T., Bari, V., Pinna, G. D., Maestri, R., and Guzzetti, S. (2012). Accounting for respiration is necessary to reliably infer granger causality from cardiovascular variability series. IEEE Trans. Biomed. Eng. 59, 832-841. doi: 10.1109/TBME.2011.2180379

Porta, A., D’addio, G., Guzzetti, S., Lucini, D., and Pagani, M. (2004). "Testing the presence of non stationarities in short heart rate variability series," in Computers in Cardiology, 2004 (Chicago, IL: IEEE), 645-648.

Radaelli, A., Bernardi, L., Valle, F., Leuzzi, S., Salvucci, F., Pedrotti, L., et al. (1994). Cardiovascular autonomic modulation in essential hypertension. effect of tilting. Hypertension 24, 556-563. doi: 10.1161/01.HYP.24. 5.556

Reimann, M., Friedrich, C., Gasch, J., Reichmann, H., Rüdiger, H., and Ziemssen, T. (2010). Trigonometric regressive spectral analysis reliably maps dynamic changes in baroreflex sensitivity and autonomic tone: the effect of gender and age. PLoS ONE 5:e12187. doi: 10.1371/journal.pone.0012187 
Sakakibara, M., and Hayano, J. (1996). Effect of slowed respiration on cardiac parasympathetic response to threat. Psychosom. Med. 58, 32-37. doi: 10.1097/00006842-199601000-00006

SocBrasCardio (2010). Vi diretrizes brasileiras de hipertensão. Arq. Bras Cardiol. 95, 1-51. doi: 10.1590/S0066-782X2010001700001

Somers, V. K., Mark, A. L., Zavala, D. C., and Abboud, F. M. (1989a). Contrasting effects of hypoxia and hypercapnia on ventilation and sympathetic activity in humans. J. Appl. Physiol. 67, 2101-2106. doi: 10.1152/jappl.1989.67. 5.2101

Somers, V. K., Mark, A. L., Zavala, D. C., and Abboud, F. M. (1989b). Influence of ventilation and hypocapnia on sympathetic nerve responses to hypoxia in normal humans. J. Appl. Physiol. 67, 2095-2100.

Stankovski, T., Cooke, W. H., Rudas, L., Stefanovska, A., and Eckberg, D. L. (2013). Time-frequency methods and voluntary rampedfrequency breathing: a powerful combination for exploration of human neurophysiological mechanisms. J. Appl. Physiol. 115, 1806-1821. doi: 10.1152/japplphysiol.00802.2013

Stauss, H. M. (2007). Identification of blood pressure control mechanisms by power spectral analysis. Clin. Exp. Pharmacol. Physiol. 34, 362-368. doi: 10.1111/j.1440-1681.2007.04588.x

Steffen, P. R., Austin, T., DeBarros, A., and Brown, T. (2017). The impact of resonance frequency breathing on measures of heart rate variability, blood pressure, and mood. Front. Public Health 5:222. doi: 10.3389/fpubh.2017.00222

Stein, C., Dal Lago, P., Ferreira, J. B., Casali, K. R., and Plentz, R. D. M. (2011). Transcutaneous electrical nerve stimulation at different frequencies on heart rate variability in healthy subjects. Auton. Neurosci. 165, 205-208. doi: 10.1016/j.autneu.2011.07.003

Tobaldini, E., Nobili, L., Strada, S., Casali, K. R., Braghiroli, A., and Montano, N. (2013). Heart rate variability in normal and pathological sleep. Front. Physiol. 4:294. doi: 10.3389/fphys.2013.00294

Tzeng, Y., Sin, P. Y., Lucas, S. J., and Ainslie, P. N. (2009). Respiratory modulation of cardiovagal baroreflex sensitivity. J. Appl. Physiol. 107, 718-724. doi: 10.1152/japplphysiol.00548.2009

Van de Borne, P., Mezzetti, S., Montano, N., Narkiewicz, K., Degaute, J. P., and Somers, V. K. (2000). Hyperventilation alters arterial baroreflex control of heart rate and muscle sympathetic nerve activity. Am. J. Physiol. Heart Circ. Physiol. 279, H536-H541. doi: 10.1152/ajpheart.2000.279.2.H536

Conflict of Interest Statement: The authors declare that the research was conducted in the absence of any commercial or financial relationships that could be construed as a potential conflict of interest.

Copyright (C) 2018 De Souza, Ferreira, Schein, Dartora, Casali, Scassola, Tobaldini, Montano, Guzzetti, Porta, Irigoyen and Casali. This is an open-access article distributed under the terms of the Creative Commons Attribution License (CC BY).

The use, distribution or reproduction in other forums is permitted, provided the original author(s) and the copyright owner(s) are credited and that the original publication in this journal is cited, in accordance with accepted academic practice. No use, distribution or reproduction is permitted which does not comply with these terms. 